\title{
Modelagem matemática da autodepuração da microbacia do Irurá no município de Santarém - Pará
}

\section{Mathematical modeling of the self-purification of the Irurá watershed in the municipality of Santarém - Pará}

Modelación matemática de la autodepuración de la
microcuenca del Irurá en el municipio de Santarém - Pará

\begin{abstract}
Modélisation mathématique de l'auto-purification du micro bassin versant d'Irurá dans la municipalité de Santarém, dans l'état du Pará, au Brésil
\end{abstract}

\section{Resumo}

Raquel Freitas dos Santos i freitasbesa@gmail.com Universidade Federal do Oeste do Pará (Ufopa)

Zaqueu dos Santos iD zaqueu_sant@hotmail.com Universidade Federal do Oeste do Pará (Ufopa)

Mirian Santos de Sousa iD miriansantos1203@gmail.com Universidade Federal do Oeste do Pará (Ufopa)

Ruy Bessa Lopes (iD ruybessa@yahoo.com.br Universidade Federal do Oeste do Pará (Ufopa)

Lucinewton Silva de Moura id lucinewton.moura@ufopa.edu.br Universidade Federal do Oeste do Pará (Ufopa)
Os ecossistemas aquáticos possuem um mecanismo natural de recuperação após receberem uma determinada carga de poluentes, e tal capacidade de autodepuração pode ser analisada através da modelagem matemática. No município de Santarém, muitos corpos d'água sofrem com a crescente degradação, tornando-se necessário monitorar e avaliar a qualidade da água da microbacia e buscar respostas através da modelagem, avaliando a sua capacidade de autodepuração. O objetivo do presente estudo foi avaliar a capacidade de autodepuração da microbacia do Irurá, de um corpo d'água urbano, através da análise da autodepuração do oxigênio dissolvido (OD) utilizando modelagem matemática. A coleta de dados ocorreu nos anos de 2016 e 2017. Para o ajuste dos coeficientes da modelagem matemática foi utilizado o software Curver Expert 1.4, usando o método matemático de ajuste de funções não lineares de Levenberg-Marquardt. Foi inserido o modelo matemático de Streeter-Phelps completo levando em consideração as parcelas de déficit pontual, DBO pontual, nitrificação, demanda de sedimento, fotossíntese e respiração. Foram ajustados oito coeficientes do modelo em função dos dados experimentais de oxigênio dissolvido e do tempo de percurso d'água. Diferentemente dos outros trabalhos apresentados na literatura, os coeficientes foram obtidos através de ajuste do modelo matemático de Streeter-Phelps. A partir dos coeficientes ajustados foi possível predizer o nível de decaimento do OD a qualquer tempo de percurso do corpo d'água, possibilitando a identificação das seções com nível crítico de OD.

Palavras-chave: Autodepuração. Oxigênio dissolvido. Modelagem. 
model coefficients were adjusted according to the experimental data of dissolved oxygen and water travel time. Unlike the other studies presented in the literature, the coefficients were obtained by adjusting the Streeter-Phelps mathematical model. From the adjusted coefficients, it was possible to predict the level of decay of the OD at any time of the water body, thus enabling the identification of the sections with a critical level of OD.

Keywords: Self-purification. Dissolved oxygen. Modeling.

\section{Resumen}

Los ecosistemas acuáticos poseen un mecanismo natural de recuperación después de recibir una determinada carga de contaminantes. Esta capacidad de autodepuración puede ser analizada por medio de la modelación matemática. En el municipio de Santarém, muchos cuerpos de agua sufren con creciente degradación, haciéndose necesario monitorear y evaluar la calidad del agua de la microcuenca y buscar respuestas por medio de la modelación, evaluando su capacidad de autodepuración. El objetivo de este trabajo fue evaluar la capacidad de autodepuración de la microcuenca del Irurá, de un cuerpo de agua urbano, por medio del análisis de la autodepuración del oxigeno disuelto (OD) utilizando la modelación matemática. La recogida de datos ocurrió en los años de 2016 y 2017. Para el ajuste de los coeficientes de la modelación matemática se utilizó el software Curver Expert 1.4, utilizando el método matemático de ajuste de función no linear de LevenbergMarquardt. Fue inserido el modelo matemático de Streeter-Phelps completo llevando en consideración las parcelas del déficit puntual, DBO puntual, nitrificación, demanda de sedimento, fotosíntesis y respiración. Fueron ajustados ocho coeficientes del modelo en función de los datos experimentales de oxigeno disuelto y del tiempo de recorrido del agua. Diferentemente de los otros trabajos presentados en la literatura, en este los coeficientes fueron obtenidos por medio de ajustes del modelo matemático de Streeter-Phelps. A partir de los coeficientes ajustados fue posible predecir el nivel de decaimiento del OD en cualquier tiempo del recorrido del cuerpo de agua, posibilitando así la identificación de las secciones con nivel crítico de OD.

Palabras-clave: Autodepuración. Oxígeno disuelto. Modelación.

\section{Résumé}

Les écosystèmes aquatiques ont un mécanisme de récupération naturel après avoir reçu une certaine charge de polluants, une telle capacité d'auto-purification peut être analysée par le biais d'une modélisation mathématique. Dans la municipalité de Santarém, nombreux plans d'eau souffrent d'une dégradation croissante. Donc, il faut surveiller et évaluer la qualité du bassin versant et chercher des réponses par moyen de la modélisation, en évaluant sa capacité d'auto-nettoyage. L'objectif de la présente étude a été d'évaluer la capacité d'auto-nettoyage du micro bassin versant d'Irurá, d'un plan d'eau urbain, à travers l'analyse de l'auto-nettoyage de l'oxygène dissous (OD) à l'aide d'une modélisation mathématique. La collecte de données a eu lieu entre 2016 et 2017. Pour l'ajustement des coefficients de la modélisation mathématique, le logiciel Curver Expert 1.4 a été utilisé, en utilisant la méthode mathématique d'ajustement des fonctions non linéaires de Levenberg-Marquardt. Le modèle mathématique complet de Streeter-Phelps a été inséré en tenant compte des portions du déficit ponctuel, de la DBO ponctuelle, de la nitrification, de la demande en sédiments, de la photosynthèse et de la respiration. Huit coefficients du modèle ont été ajustés en fonction des données expérimentales de l'oxygène dissous et du temps de parcours de l'eau. Contrairement à d'autres travaux présentés dans la littérature, dans ce cas, les coefficients ont été obtenus en ajustant le modèle mathématique de Streeter-Phelps. À partir des coefficients ajustés, il a été possible de prédire le niveau de décroissance de l'OD à tout moment où le plan d'eau se déplace. Ce qui permet, ainsi, d'identifier les sections avec un niveau d'OD critique.

Mots-clés: auto-nettoyage. Oxygène dissous. Modelage. 


\section{Introdução}

Devido ao crescimento populacional humano ao longo dos séculos, observa-se que as atividades antrópicas influenciam direta e/ou indiretamente os ecossistemas e, consequentemente, na perda da biodiversidade. A busca pelo crescimento econômico tem pressionado os recursos naturais para obtenção de bens e serviços e, nesse processo, tem produzido contaminação e poluição para o meio ambiente (GOULART; CALLISTO, 2003). O Brasil apresenta uma extensa e densa rede hidrográfica, que têm recebido descargas de poluentes em seus corpos hídricos, contribuindo para sua degradação (BARRETO, 2009) e influenciando a qualidade de vida humana, o desenvolvimento econômico e os sistemas naturais (TUNDISI, 2003).

Naturalmente, os ambientes aquáticos possuem um mecanismo de recuperação após a entrada de fontes poluidoras, um fenômeno de sucessão ecológica denominado de autodepuração. A habilidade de autodepuração se modifica de um corpo hídrico para outro, tornando-se indispensável o desenvolvimento de estudos específicos com a finalidade de conhecer a carga de efluentes que o rio é capaz de receber e diluir sem que suas características naturais sejam prejudicadas. A modelagem matemática da qualidade da água é uma ferramenta utilizada para verificar a capacidade de autodepuração de rios. Através de processos físicos, químicos e biológicos, possibilitam a análise de valores de seus parâmetros que influenciam na entrada e no consumo de oxigênio do corpo d'água (VON SPERLING, 2005).

A modelagem matemática é uma ferramenta que está presente na sociedade e abrange as mais diversas áreas do conhecimento. É uma maneira de estudar os sistemas do mundo real, seus fenômenos físicos, biológicos, sociais, psicológicos, conceituais, ou, até mesmo, outro modelo matemático, e transformá-los em modelos que os representam de maneira simplificada, havendo assim uma melhor compreensão desses sistemas (FRAGOSO et al., 2009; SODRÉ, 2007; BASSANEZI, 1999).

O ecossistema aquático, por ter alta complexidade e envolver diferentes variáveis, é um campo fértil de aplicações de instrumentos que simplifiquem e exemplifiquem tais relações ambientais. Sendo assim, os modelos matemáticos apresentam-se como uma ferramenta viável economicamente e de poucas incertezas no entendimento da dinâmica hidrobiológica de um corpo receptor. Permite diagnosticar o estado atual de determinado corpo hídrico e como ele poderá se comportar ao receber cargas de poluentes, servindo de base para a tomada de decisão que controle ou reduza o lançamento de poluentes sobre os ecossistemas aquáticos (FRAGOSO et al., 2009).

Entre os modelos matemáticos de qualidade da água, observa-se o modelo de Streeter-Phelps, o qual descreve o aumento e decréscimo do déficit de oxigênio em um corpo hídrico que recebe uma fonte de material orgânico. Esse modelo foi aplicado, em 1925, no Rio Ohio, com o objetivo de contribuir para que as ações de controle da poluição fossem mais eficientes. Foi o pioneiro de outros modelos atuais de qualidade da água, tais como QUAL I e II QUAL-2E e QUAL-2K (RICCIARDONE et al., 2011).

Diante disso, o objetivo do presente estudo foi avaliar a capacidade de autodepuração da microbacia do Irurá, de um corpo d'água urbano, através da análise da autodepuração do oxigênio dissolvido (OD), utilizando modelagem matemática.

\section{Coeficiente de desoxigenação $\left(\mathrm{K}_{1}\right)$}

O coeficiente de desoxigenação depende de variáveis como o tipo de matéria orgânica, temperatura, substâncias inibidoras, além do grau de tratamento. Para o seu cálculo é necessário que se conheça a DBO última (VON SPERLING, 2005).

No modelo de Streeter-Phelps, o consumo de DBO segue uma reação de primeira ordem, na qual a taxa de redução da matéria orgânica é proporcional à sua concentração em um determinado instante t (VON SPERLING, 2014). De acordo com a Eq. (1).

$$
L_{0} \cdot e^{-K_{1} \cdot t}
$$


2. Coeficiente de reaeração $\left(\mathrm{K}_{2}\right)$

O coeficiente de reaeração pode ser estimado, a partir de três procedimentos, para que possa simular o OD no curso d'água estudado. São eles: valores médios tabelados; valores relacionados às características hidráulicas e morfológicas do rio, e valores obtidos em função da vazão do curso d'água (VON SPERLING, 2005).

A cinética de reaeração pode ser representada pela seguinte Eq. (2):

$$
\frac{\mathrm{dD}}{d t}=-K_{2} \cdot D
$$

Equação diferencial do déficit de OD Eq. (3):

$$
\frac{d L}{d t}=-K_{2} \cdot\left(C_{s}-C\right)+K_{d} \cdot L+S_{d}-F+R+R O 2 a m o n \cdot\left(f_{n i t r} \cdot K_{a n}\right) \cdot N_{a m o n}
$$

Equação integrada analiticamente do déficit de OD Eq. (4):

$$
\begin{aligned}
& D=\quad D_{0} \cdot e^{-K_{2} \cdot t} \quad \text { Déficit pontual }
\end{aligned}
$$

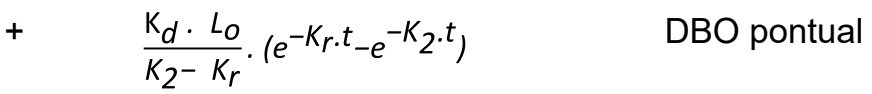

$$
\begin{aligned}
& +\frac{R_{\text {O2amon }} \cdot K_{a n} \cdot N_{a m o n 0}}{K_{2}-K_{a n}} \cdot\left(e^{-K_{a n} \cdot t_{-}} e^{-K_{2} \cdot t_{t}}\right) \quad \text { Nitrificação } \\
& +S_{d} \cdot\left(1-e^{-K_{2} \cdot t}\right) \quad \text { Demanda de Sedimentos } \\
& +\quad \frac{F}{K_{2}} \cdot\left(1-e^{-K_{2} \cdot t}\right) \quad \text { Fotossíntese } \\
& +\frac{R}{K_{2}} \cdot\left(1-e^{-K_{2} \cdot t_{1}} \quad\right. \text { Respiração }
\end{aligned}
$$

\section{Modelo QUAL-2E}

Desde a criação do modelo de Streeter-Phelps (1925), diversos modelos da qualidade da água foram surgindo no decorrer do tempo; modelos mais completos e complexos, que envolvem diversas variáveis, com o objetivo de representar os ecossistemas aquáticos de maneira mais precisa. E, entre esses modelos, pode-se incluir o QUAL-2E (1987).

O QUAL-2E foi propagado e distribuído pela Environmental Protection Agency - USEPA (Agência de Proteção dos Estados Unidos). Muito utilizado atualmente, é um modelo que considera os ciclos de O, N e P na água de maneira mais intensa (VON SPERLING, 2014).

\section{4. $\quad$ Modelo QUAL UFMG}

O QUAL UFMG é um modelo matemático proposto por Von Sperling. É baseado no modelo QUAL-2K (USEPA), no entanto, sem a modelagem aplicada às algas e sem considerar a dispersão longitudinal. Com o QUAL UFMG é possível simular a reposição de OD que foi consumido em um corpo hídrico, reaeração, bem como seus efeitos sobre o balanço desse gás. Ele simula também o decaimento da matéria orgânica, a presença de coliformes e séries do nitrogênio e fósforo (TEODORO, 2013). 


\section{Metodologia}

\section{1 Área de estudo}

O estudo foi realizado na microbacia Irurá, pertence à bacia hidrográfica do Rio Tapajós, localizada no município de Santarém, estado do Pará, entre as coordenadas geográficas: longitude $054^{\circ} 45^{\prime} 13,8^{\prime \prime}$ W e $054^{\circ}$ 44' 09.2" e latitude $02^{\circ} 29^{\prime} 49,3^{\prime \prime}$ e $02^{\circ} 26^{\prime} 29.6^{\prime \prime} \mathrm{S}$ (Figura 1), abrangendo os bairros Cambuquira, Matinha, Maracanã, Mapiri e Santarenzinho. O igarapé possui uma extensão de, aproximadamente, 9,2905 Km da sua nascente até sua foz, no Rio Tapajós. A cobertura vegetal às margens do curso d'água é predominante na maior parte de sua extensão, exceto em áreas onde há influência de estradas, pontes, áreas particulares e na proximidade do perímetro urbano.

Figura 1 - Localização geográfica da microbacia hidrográfica do Igarapé Irurá.

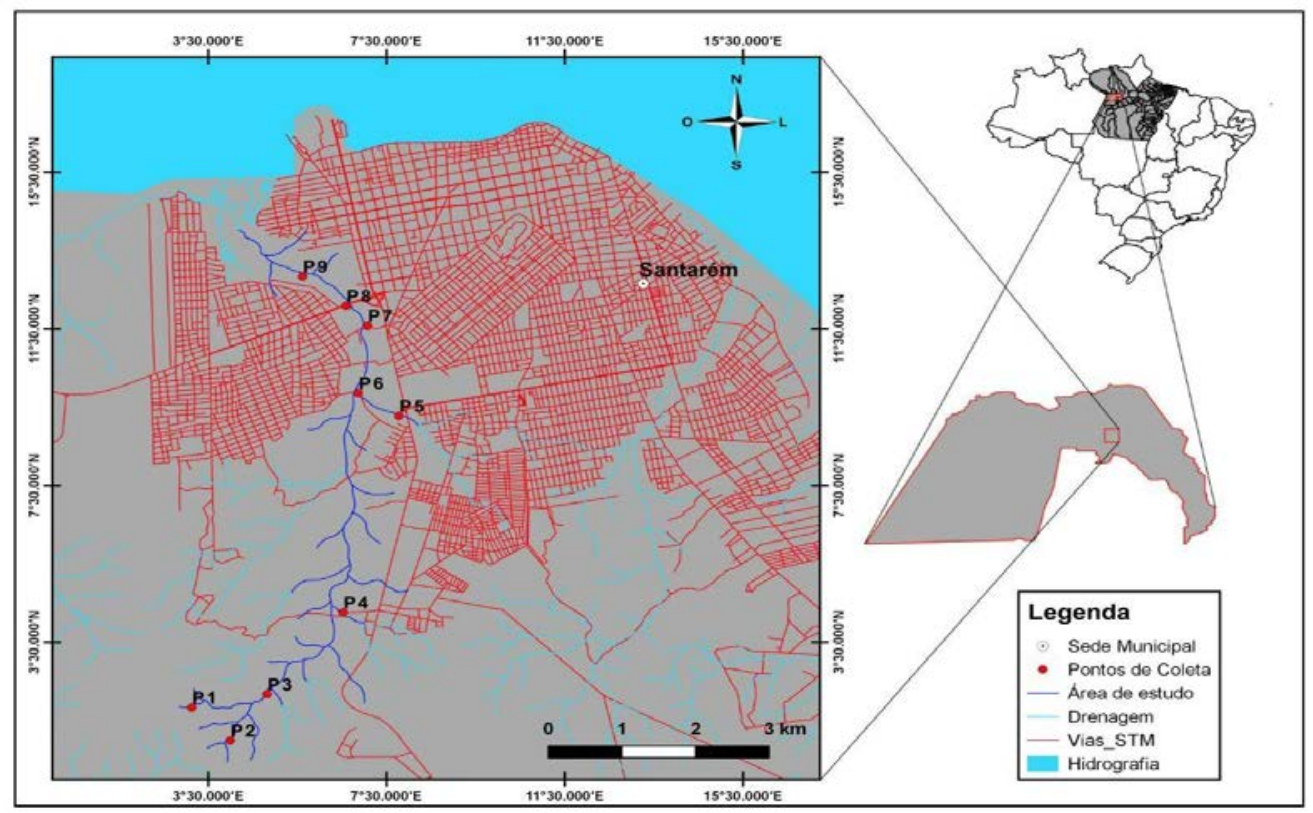

Fonte: Autor, 2017.

O município de Santarém localiza-se na região do Baixo Amazonas, no estado do Pará, sendo pertencente ao território da Amazônia Legal. O tipo climático da região é Am, segundo a classificação de Kõppen, ou seja, pertence ao domínio do clima tropical, apresentando alta pluviosidade $(2.000 \mathrm{~mm})$ e um razoável período de estiagem. As temperaturas anuais da região oscilam entre $25^{\circ}$ e $26^{\circ} \mathrm{C}$ (médias), $30^{\circ}$ e $31^{\circ} \mathrm{C}$ (máximas) e $21^{\circ}$ e $23^{\circ} \mathrm{C}$ (mínimas) (ALVARES et al., 2013; TSUKAMOTO FILHO, et al., 2007).

\subsection{Procedimento de coleta}

As amostragens do oxigênio dissolvido foram realizadas em nove seções de coleta nos períodos: chuvoso (outubro e novembro de 2016) e menos chuvoso (maio e junho de 2017). Os ensaios foram medidos em quadruplicata. As seções amostrais cobriram a nascente e a montante do igarapé. $O$ critério de eleição dos pontos a jusante do igarapé se relacionou estritamente com a maior intensidade no uso e ocupação do solo ao longo do corpo receptor. Os métodos de coleta e análise da água do Igarapé Irurá foram baseados na NBR 9897, de junho 1987, que descreve o planejamento de amostras e efluentes líquidos e corpos receptores (ABNT, 1987). O oxigênio dissolvido foi analisado in locu através de oxímetro (55 YSI), com capacidade de medição de 0 a 20 mg.L-1 e método Galvânico, Clark (níquel/chumbo). A vazão foi determinada através do equipamento Flow Tracker, número de série P-3260, fabricado pela Sontek/YSI. 


\subsection{Equações do modelo}

Para o cálculo da concentração de OD é utilizada a Eq. (5):

Sabendo que:

$$
C_{t}=C_{s}-D_{t}
$$

Logo, a concentração de OD no tempo t é obtida na Eq.( 6):

$$
\mathrm{C}_{\mathrm{t}}=\mathrm{C}_{5}-\left[\frac{\mathrm{K}_{1} \cdot \mathrm{L}_{0}}{\mathrm{~K}_{2} \cdot \mathrm{K}_{1}}\left(\mathrm{e}^{-\mathrm{K}_{1} \cdot \mathrm{t}}-\mathrm{e}^{-\mathrm{K}_{2} \cdot \mathrm{t}}\right)+\left(\mathrm{C}_{5}-\mathrm{C}_{0}\right) \cdot \mathrm{e}^{-\mathrm{K}_{2} \cdot \mathrm{t}}\right]
$$

Onde:

$\mathrm{C}_{\mathrm{t}}=$ concentração de OD no tempo $\mathrm{t}\left(\mathrm{mg} \cdot \mathrm{L}^{-1}\right)$;

$\mathrm{C}_{\mathrm{s}}=$ concentração de saturação de oxigênio $\left(\mathrm{mg} \cdot \mathrm{L}^{-1}\right)$;

$\mathrm{C}_{\mathrm{o}}=$ concentração inicial de oxigênio logo após a mistura $\left(\mathrm{mg} \cdot \mathrm{L}^{-1}\right)$;

$\mathrm{K}_{1}=$ coeficiente da taxa de desoxigenação $\left(\mathrm{dia}^{-1}\right)$;

$\mathrm{K}_{2}=$ coeficiente da taxa de reaeração $\left(\right.$ dia $\left.^{-1}\right)$;

$\mathrm{L}_{\mathrm{o}}=$ concentração de determinado poluente, no corpo receptor, após a mistura com o despejo (mg. $\left.\mathrm{L}^{-1}\right)$;

$\mathrm{D}_{\mathrm{t}}=$ déficit inicial de OD no ponto da mistura $\left(\mathrm{mg} \cdot \mathrm{L}^{-1}\right)$.

\subsection{Calibração do modelo}

A calibração de um modelo matemático de qualidade da água consiste em adequar os parâmetros da equação ao mundo real, considerando as variáveis físicas, químicas e biológicas desse ambiente, ajustando os dados estimados na modelagem com os dados observados em campo (TONON et al., 2014). Para a calibração do modelo matemático apresentado neste trabalho, utilizaram-se dados experimentais de tempo de percurso (t) e oxigênio dissolvido (OD).

O tempo de percurso foi transformado em dias através da Eq. (7), em cada uma das seções de coleta, em função da distância percorrida e da velocidade do curso d'água, considerando valores médios de velocidade ao longo do percurso d'água.

A velocidade foi determinada em cada seção de coleta utilizando o equipamento Flow Tracker, descrito no item 2.2, e o seu valor médio no percurso d'agua utilizada para o cálculo do tempo, de $0,28 \mathrm{~m} . \mathrm{s}^{-1}$.

$$
\mathrm{t}=\frac{d}{v .86400}
$$

Onde:

$\mathrm{t}=$ tempo de percurso (dia);

$\mathrm{d}=$ distância percorrida em $(\mathrm{m})$;

$\mathrm{v}=$ velocidade do curso d'água $\left(\mathrm{m} \cdot \mathrm{s}^{-1}\right)$;

$86400=$ número adimensional de conversão de segundo para dia $\left(\mathrm{s}_{\mathrm{g}} \mathrm{dia}^{-1}\right)$.

\subsection{Método de ajuste dos coeficientes do modelo de Streeter-Phelps (1925)}

A metodologia para a obtenção dos oito parâmetros ajustáveis da Eq. (6) consistiu em utilizar os dados dos valores médios experimentais do oxigênio dissolvido (OD) e do tempo de percurso do fluxo de água (t) desde a seção um (nascente do igarapé) até a seção nove (foz).

Os oito coeficientes $\left(L_{0}, K_{2}, S_{d}, F, R, \alpha, K_{a n}\right.$ e $\left.K_{r}\right)$ foram ajustados a partir do software Curv Expert, versão 1.4 , utilizando o método matemático de ajuste de funções não lineares de Levenberg-Marquardt. $O$ método 
matemático de ajuste de funções não lineares de Levenberg-Marquardt necessita de uma estimativa inicial dos seus parâmetros a serem ajustados para iniciar o processo interativo de ajuste de uma função. Foram atribuídos como estimativa inicial, de cada um dos oito coeficientes ajustáveis, valores médios encontrados em VON SPERLING (2014). O procedimento de ajuste consistiu em uma estimativa inicial para cada um dos oito coeficientes, até ajustá-los, utilizando o maior valor do coeficiente de correlação $R_{2}$ como critério de tomada de decisão para a melhor otimização desses coeficientes.

Os valores experimentais da concentração de oxigênio na saturação e do déficit de oxigênio inicial foram, respectivamente, $8,06 \mathrm{mg} / \mathrm{L}$ e $0,46 \mathrm{mg} / \mathrm{L}$. Foi utilizado como coeficiente de entrada somente o valor do coeficiente de decomposição $K_{d}$, igual a 0,45, obtido a partir de valores médios encontrados em VON SPERLING (2014) (nas condições de similaridade com o igarapé estudado). $O \mathrm{~K}_{\mathrm{d}}$ foi arbitrariamente utilizado como único coeficiente de entrada em razão da dificuldade de ajustá-lo, simultaneamente, com $L_{0}$, uma vez que, na Eq. (6), esses dois termos são multiplicados um pelo outro, o que impossibilita o ajuste simultâneo desses dois coeficientes. Por esse motivo, decidimos optar pelo ajuste do $\mathrm{L}_{0}$. $\mathrm{O}$ mesmo pode ser evidenciado na parcela de nitrificação também na Equação 6, onde temos o termo ( $\alpha=\mathrm{R}_{\mathrm{Ozamon}} \cdot \mathrm{K}_{\mathrm{an}} \cdot \mathrm{N}_{\text {amono }}$ ). Nesse caso específico utilizamos como coeficiente de ajuste $\alpha$ e $\mathrm{K}_{a n}$, no entanto o valor do coeficiente $\mathrm{N}_{\text {amono }}$ foi determinado indiretamente a partir do conhecimento do $\mathrm{K}_{\mathrm{an}}$ e $\alpha$ (ajustado aos dados experimentais) e do valor médio de $\mathrm{R}_{\mathrm{O} 2 a m o n}$ igual a 4,15, obtido em VON SPERLING (2014). O valor do coeficiente K foi determinado indiretamente a partir do valor do $\mathrm{K}_{d}$ (valor médio obtido em VON SPERLING, 2014) e do $\mathrm{K}_{\mathrm{r}}$ (ajustado aos dados experimentais).

\section{Resultados e discussão}

\subsection{Modelagem matemática}

Na Tabela (1) estão apresentadas as localizações geográficas e a descrição das nove seções de coleta ao longo da extensão da microbacia do Irurá e a distância ente as seções. Para a seleção dos locais de coleta no igarapé, levou-se em consideração a influência antrópica, a busca por uma distribuição que abrangesse toda a extensão do corpo d'água e a facilidade de acesso a essas seções. A distância total entre a primeira e última seção de coleta foi de 9,2905 Km.

Tabela 1 - Identificação das seções de amostragem realizadas na microbacia do Irurá.

\begin{tabular}{ccccc}
\hline Seção de coleta & Distância (km) & Altimetria $(\mathbf{m})$ & Latitude $(\mathbf{S})$ & Longitude $(\mathbf{W})$ \\
\hline Seção 1 & 0 & 87,2 & $02^{\circ} 29^{\prime} 49.3^{\prime \prime}$ & $054^{\circ} 45^{\prime} 13,8^{\prime \prime}$ \\
Seção 2 & 0,7220 & 73,2 & $02^{\circ} 30^{\prime} 06.7^{\prime \prime}$ & $054^{\circ} 45^{\prime} 0.07^{\prime \prime}$ \\
Seção 3 & 1,6235 & 58,5 & $02^{\circ} 29^{\prime} 43.7^{\prime \prime}$ & $054^{\circ} 44^{\prime} 43.9^{\prime \prime}$ \\
Seção 4 & 3,2705 & 52 & $02^{\circ} 29^{\prime} 02.8^{\prime \prime}$ & $054^{\circ} 44^{\prime} 10.5^{\prime \prime}$ \\
Seção 5 & 6,4045 & 55 & $02^{\circ} 27^{\prime} 24.6^{\prime \prime}$ & $054^{\circ} 43^{\prime} 46.0^{\prime \prime}$ \\
Seção 6 & 7,0705 & 27 & $02^{\circ} 27^{\prime} 13.2^{\prime \prime}$ & $054^{\circ} 44^{\prime} 03.8^{\prime \prime}$ \\
Seção 7 & 8,1255 & 16 & $02^{\circ} 26^{\prime} 39.5^{\prime \prime}$ & $054^{\circ} 43^{\prime} 59.8^{\prime \prime}$ \\
Seção 8 & 8,5475 & 19 & $02^{\circ} 26^{\prime} 29.6^{\prime \prime}$ & $054^{\circ} 44^{\prime} 09.2^{\prime \prime}$ \\
Seção 9 & 9,2905 & 42 & $02^{\circ} 26^{\prime} 14.9^{\prime \prime}$ & $054^{\circ} 44^{\prime} 28.3^{\prime \prime}$ \\
\hline
\end{tabular}

Destaca-se, nesse trabalho, um diferencial em relação aos demais trabalhos apresentados recentemente (STRAGER et al., 2010; CUNHA et al., 2017; HOLANDA et al., 2017; GOMES et al., 2018; GONÇALVES; ESPOSITO, 2019; YUSTIANI et al., 2019; WAGHMADE; KIWNE, 2019). Os coeficientes são ajustados aos dados experimentais de OD versus tempo de percurso, além de ser utilizada a equação de Streeter-Phelps completa, levando em consideração todas as parcelas: déficit pontual, DBO pontual, nitrificação, demanda de sedimento, fotossíntese e respiração, o que minimiza o erro da predição, uma vez que se leva em consideração as condições ambientais regionais. 
Outro aspecto relevante a ser considerado é que, neste trabalho, a partir dos coeficientes ajustados, é possível estimar a qualquer tempo do percurso do corpo d'água o nível de decaimento do oxigênio dissolvido ao longo do percurso do igarapé, possibilitando diagnosticar o grau de comprometimento do corpo hídrico e dando subsídios necessários para os órgãos ambientais nas tomadas de decisão e de medidas mitigadoras de intervenção para recuperação do manancial.

Na Tabela (2) estão apresentados os valores dos oitos coeficientes do modelo matemático de StreeterPhelps, Eq. (6), ajustados a partir dos dados experimentais do tempo de percurso versus OD.

Tabela 2 - Coeficientes do modelo matemático de Streeter-Phelps ajustados aos dados experimentais do tempo de percurso/OD.

\begin{tabular}{lc}
\hline Coeficientes & $\begin{array}{c}\text { Valores ajustados } \\
\text { (adimensional) }\end{array}$ \\
\hline $\mathrm{L} 0$ & 62,21 \\
$\mathrm{~K}_{2}$ & 1,28 \\
$\mathrm{Sd}$ & 4,13 \\
$\mathrm{~F}$ & 0,039 \\
$\mathrm{R}$ & 4,46 \\
$\alpha\left(\mathrm{RO}_{2}\right.$ amon.Kan.Namon) onde: Namon=3,66; Ro ${ }_{2}$ media literatura=4,15 & 2,96 \\
$\mathrm{Kan}$ & 0,20 \\
$\mathrm{~K}_{\mathrm{r}}\left(\mathrm{K}_{\mathrm{r}}=\mathrm{K}_{\mathrm{S}}+\mathrm{K}_{\mathrm{d}}\right)$ & 1,43 \\
\hline
\end{tabular}

Na Tabela (3) estão apresentados os dados calculados para o déficit de OD e as contribuições de cada parcela de déficit e DBO pontual, nitrificação, demanda de sedimentação, fotossíntese e respiração através da Eq.(6).

Tabela 3 - Dados experimentais de tempo de percurso/OD e dados calculados a partir da equação completa de Streeter-Phelps utilizando os coeficientes ajustados aos dados experimentais do tempo de percurso/OD.

\begin{tabular}{|c|c|c|c|c|c|c|c|c|}
\hline $\begin{array}{l}\text { Seção de } \\
\text { coleta }\end{array}$ & $\begin{array}{c}\text { Tempo } \\
\left(\mathbf{d}^{-1}\right)\end{array}$ & $\begin{array}{c}\text { Déficit de } \\
\text { OD } \\
\text { exp. } \\
\text { (mg/L) }\end{array}$ & $\begin{array}{c}\text { Déficit } \\
\text { e DBO } \\
\text { pontual } \\
\text { calc. } \\
\text { (mg/L) }\end{array}$ & $\begin{array}{l}\text { Nitrificação } \\
\text { calc. } \\
\text { (mg/L) }\end{array}$ & $\begin{array}{c}\text { Demanda de } \\
\text { sedimentação } \\
\text { cal. } \\
(\mathrm{mg} / \mathrm{L})\end{array}$ & $\begin{array}{c}\text { Fotossíntese } \\
\text { calc. } \\
\text { (mg/L) }\end{array}$ & $\begin{array}{l}\text { Respiração } \\
\text { calc. (mg/L) }\end{array}$ & $\begin{array}{l}\text { Déficit } \\
\text { OD } \\
\text { calc. } \\
\text { (mg/L) }\end{array}$ \\
\hline Seção 1 & 0 & $7,60 \pm 0,02$ & 0,4600 & 0,000 & 0,000 & 0,0000 & 0,0000 & 7,60 \\
\hline Seção 2 & 0,03 & $7,70 \pm 0,04$ & 1,2492 & 0,087 & 0,155 & 0,0012 & 0,1313 & 7,18 \\
\hline Seção 3 & 0,067 & $6,90 \pm 0,22$ & 2,1353 & 0,189 & 0,338 & 0,0025 & 0,2864 & 6,74 \\
\hline Seção 4 & 0,136 & $5,60 \pm 0,14$ & 3,5536 & 0,365 & 0,658 & 0,0049 & 0,5569 & 6,08 \\
\hline Seção 5 & 0,266 & $5,41 \pm 0,01$ & 5,5218 & 0,650 & 1,189 & 0,0088 & 1,0060 & 5,37 \\
\hline Seção 6 & 0,293 & $5,64 \pm 0,14$ & 5,8325 & 0,703 & 1,288 & 0,0096 & 1,0903 & 5,30 \\
\hline Seção 7 & 0,337 & $4,91 \pm 0,12$ & 6,2767 & 0,784 & 1,443 & 0,0107 & 1,2216 & 5,22 \\
\hline Seção 8 & 0,355 & $5,40 \pm 0,08$ & 6,4376 & 0,815 & 1,504 & 0,0112 & 1,2732 & 5,20 \\
\hline Seção 9 & 0,385 & $5,10 \pm 0,18$ & 6,6806 & 0,866 & 1,603 & 0,0119 & 1,3566 & 5,19 \\
\hline
\end{tabular}

Na Figura (2) é apresentado o gráfico do déficit de OD experimental obtido a partir dos dados experimentais do tempo de percurso d'água versus oxigênio dissolvido e o gráfico do déficit de OD calculado. $O$ gráfico do déficit de OD calculado foi obtido através da equação 6 , utilizando os coeficientes apresentados na Tab. (2).

$\mathrm{Na}$ Figura (2) observa-se, entre as seções 5 e 6, a maior influência antrópica destacada, principalmente, pela autodepuração do OD. 
Figura 2 - Perfil do OD ao longo do curso d'água do igarapé Irurá.

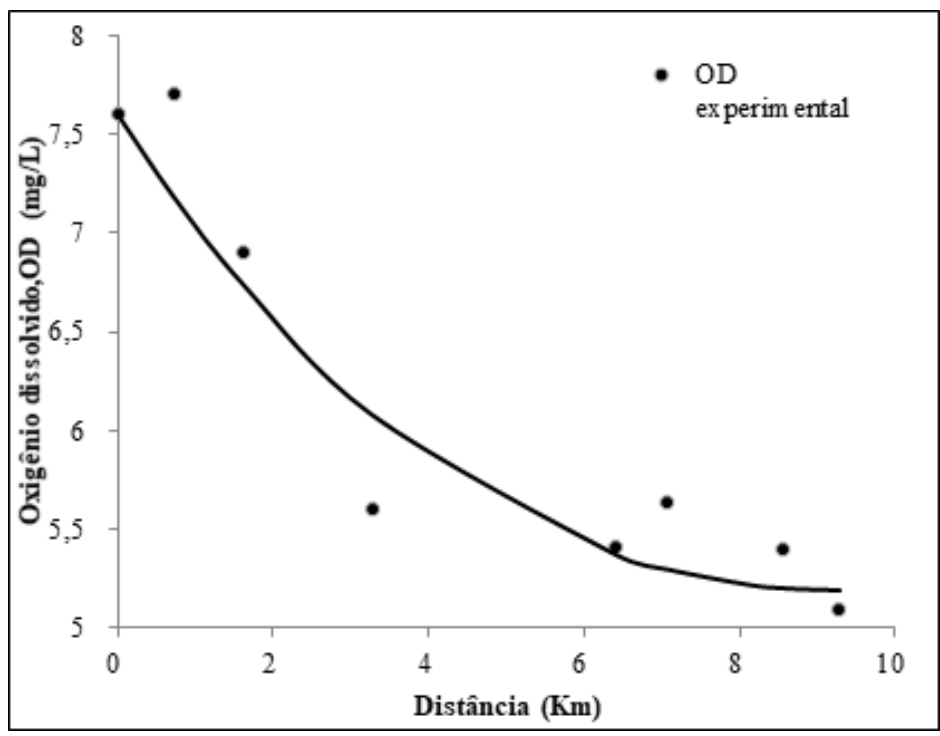

A contribuição das parcelas de déficit pontual, nitrificação, demanda de sedimentos, fotossíntese e respiração para o déficit de OD no Irurá, obtidos na modelagem matemática, pode ser observado na Fig. (3).

Figura 3 - Déficit de oxigênio longo do curso d'água Irurá.

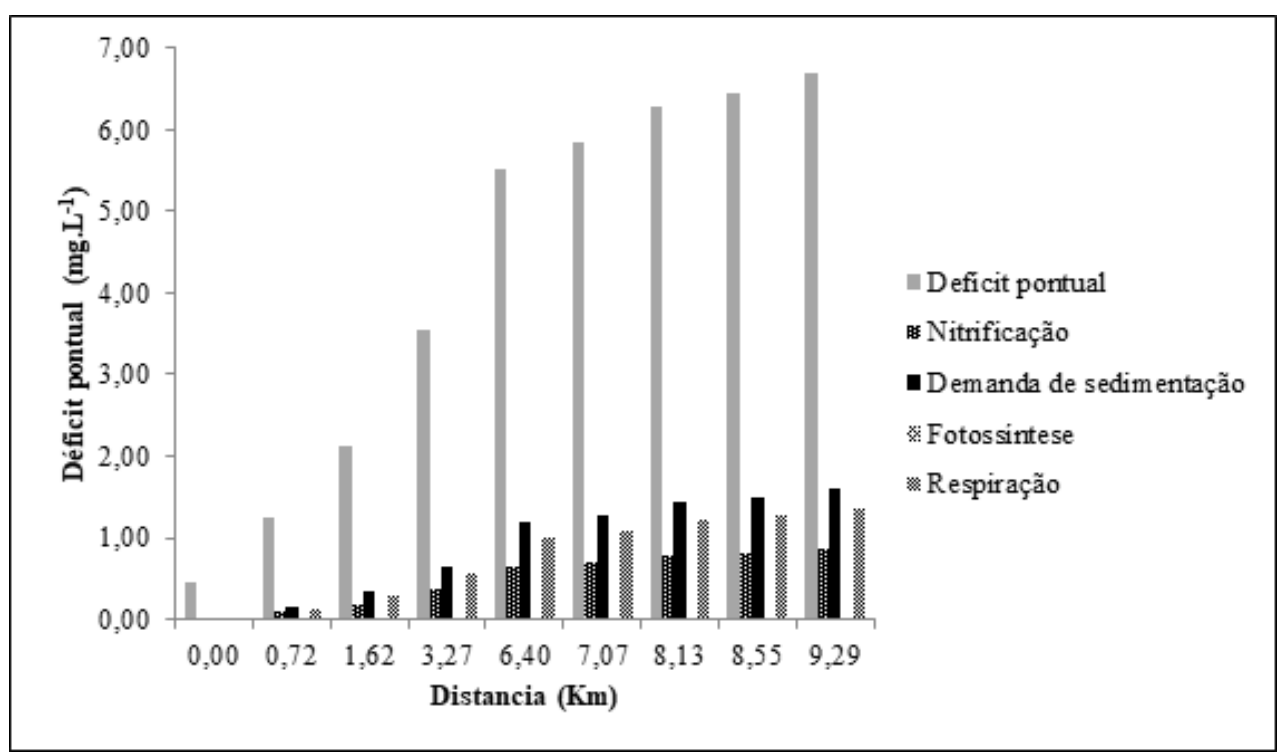

O déficit de oxigênio dissolvido $(\mathrm{OD})$ refere-se à diferença entre a concentração de saturação e concentração em um determinado momento t. Observa-se na Fig. (3) que o déficit de oxigênio é crescente à medida que aumenta a distância do percurso do curso d'água. Nos pontos iniciais, o déficit pontual apresenta baixas concentrações, o que pode ser explicado por fatores que contribuem para o aumento do OD, como a existência de maior reaeração atmosférica através da difusão turbulenta, criando-se mais interfaces, as quais se renovam permitindo uma maior absorção do oxigênio pela massa líquida (WANG et al., 1978; CHAPRA; RUNKEL, 1999; NAS; NAS, 2009; ERTURK et al., 2010; OMOLE; LONGE, 2012; VON SPERLING, 2014; BAl et al., 2019; HUANG et al., 2019), o que pode ser observado nos locais de coleta: presença de geradores de turbulências, como corredeiras, quedas d'água, alta declividade e barragens, além de o igarapé apresentar baixa profundidade. No decorrer do percurso, o déficit pontual eleva-se, possivelmente devido à ausência de fatores que contribuam 
para a absorção de OD através da difusão turbulenta, como o consumo de OD por decompositores da matéria orgânica oriunda de lançamentos difusos de esgotos de domicílios e plantas industriais.

O processo de nitrificação consiste no percurso metabólico de transformação da amônia, incialmente em nitrito e, este, em nitrato, o qual indica poluição por esgotos domésticos recentes ou remotos, respectivamente, na matéria orgânica advindas de esgotos domésticos brutos, pois é predominante a presença do nitrogênio orgânico e da amônia. No igarapé Irurá, o processo de nitrificação pode ser justificado devido à presença intensa de domicílios próximos ao curso d'água nas proximidades do igarapé, o que contribui efetivamente para o lançamento de esgotos e, até mesmo, outras fontes de nitrogênio que possam ser drenadas para o igarapé através das chuvas (RAUCH et al., 1998; FAN et al., 2009; DEKISSA et al., 2004; HAIDER et al., 2013).

Por outro lado, o processo da nitrificação, como parcela de contribuição para oxigênio dissolvido, acompanha o crescimento dos demais parâmetros que colaboram para o consumo do oxigênio no igarapé (ZOPPAS, BERNARDES; MENEGUZZI, 2016), o que demonstra sua importância ao modelo matemático, pois indica um parâmetro que coopera no déficit do oxigênio.

Considerando a demanda de sedimentação estar inteiramente relacionada à turbidez, sendo influente na qualidade do rio, tal condição ambiental pode influenciar negativamente por vários atores no cenário do Igarapé do Irurá, principalmente no aumento de sedimentos para o curso d'água, como acredita Nunes (2008). Segundo Ávila (2014), existem contribuições negativas na sedimentação. O lodo de fundo é formado a partir da sedimentação da matéria orgânica em suspensão. No lodo ocorrem dois processos de estabilização: (1) de forma anaeróbia e (2) aeróbia. Devido a essa condição, ocorre a remoção da DBO e o consumo de oxigênio do corpo d'água. No gráfico, Fig. (3), verifica-se que a demanda de sedimentação calculada com os dados do $\mathrm{K}_{2}$, Sd e tempo apresenta um crescimento exponencial, podendo indicar a ocorrência de aumento na atividade de comunidade bentônica que decanta a matéria orgânica depositada no leito do igarapé FAN et al., 2009; MOTTA et al., 2010; GOTOVTSEV, 2010). As contribuições da fotossíntese para o déficit de OD apresentaram valores mínimos em todo o percurso do igarapé.

Por último, observa-se, na Figura (3), que a respiração se eleva no decorrer do curso d'água, contribuindo para o déficit de OD. Isso pode ser ocasionado pelo despejo de matéria orgânica que, em elevadas concentrações, permite a propagação de bactérias, aumentando a demanda por oxigênio em decorrência da respiração desses organismos (JUBB et al., 1998; McMARTIN; FORRESTER, 2002; ORSSATTO, 2008; LINDENSCHMIDT et al., 2009; LIU; SCAVIA, 2010; YUSTIANI et al., 2016), o que desequilibra o balanço funcional desse sistema hidrobiológico.

\section{Conclusão}

Considerando o estudo da capacidade de autodepuração da microbacia do Irurá através de uma abordagem matemática com valores de ajuste de coeficientes mais adequados, são transparentes aos dados da região estudada. Foi observado que os valores, encontrados através da predição do OD utilizando os coeficientes do modelo matemático, que foram obtidos a partir do ajuste dos dados experimentais, quando comparado com os dados experimentais de OD obtidos in loco, foram próximos, permitindo estimar cenários futuros e identificar possíveis variáveis que estão impactando diretamente para a degradação do manancial através da diminuição do OD.

O grande diferencial deste trabalho quando comparado aos outros apresentados na literatura é que utilizou o modelo matemático de Street-Phelps. Neste trabalho, além de utilizarmos a equação completa, levando em consideração todas as contribuições, também verificamos que os coeficientes do modelo são ajustados, o que não é observado em nenhum outro trabalho. Outro aspecto de extrema relevância é que é necessário o conhecimento somente dos dados experimentais do OD e do tempo de percurso do corpo d'água para obtenção do ajuste dos coeficientes e, simultaneamente, para a identificação com precisão do nível 
de comprometimento desse manancial, dando condições para os órgãos ambientais intervirem e proporem medidas mitigadoras para definição de novos diagnósticos da realidade atual.

Em relação aos resultados obtidos através da modelagem, verificou-se que o déficit de oxigênio é crescente à medida que aumenta a distância do percurso do curso d'água, ao mesmo tempo em que a concentração de OD diminui, o que ocorre também com a de demanda de sedimentação. A fotossíntese apresentou valores mínimos em todo o percurso do igarapé, e a contribuição da nitrificação para o consumo de OD apresentou valores significativos, podendo indicar lançamentos de esgoto doméstico devido à presença de residências próximas ao curso d'água e, até mesmo, outras fontes de nitrogênio, que possam ser drenadas para o igarapé através das chuvas.

\section{Referências}

ASSOCIAÇÃO BRASILEIRA DE NORMAS TÉCNICAS. ABNT NBR 9897: planejamento de amostragem de efluentes líquidos e corpos receptores: procedimento. Rio de Janeiro: ABNT, 1987.

ALVARES, C. A.; STAPE, J. L.; SENTELHAS, P. C. de; MORAES, G.; LEONARDO, J.; SPAROVEK, G. Köppen's climate classification map for Brazil. Meteorologische Zeitschrift. [S.I], v. 22, n. 6, p. 711-728, 2013.

ÁVILA, L. C. Modelagem da qualidade da água utilizando coeficientes bibliográficos e experimentais: aplicação ao Rio Vacacaí Mirim. Orientadora: Maria do Carmo Cauduro Gastaldini, 2014. 99f. Dissertação (Mestrado em Engenharia Civil) - Centro de Tecnologia, Universidade Federal de Santa Maria, 2014.

BAI, S.; REN, N.; YOU, S.; ZHAO, X.; LI, Y.; WANG, Y. Modelling the oxygen- depleting potencial and spatially differenciated effect of sewage organics in life cycle assessment for wastewater management. Science of the Total Environment. [S. I. s.n.], v. 655. p. 1071-1080, 2019.

BARRETO, L, V.; ROCHA, F, A.; OLIVEIRA, M, S. C.; Monitoramento da qualidade da água na microbacia hidrográfica do Rio Catolé, em Itapetinga-Ba. Enciclopédia Biosfera. Goiânia: Centro Científico Conhecer, v. 5, n. 8, 2009.

BASSANEZI, R. C. Modelagem matemática: uma disciplina emergente nos programas de formação de professores. Bio Matemática. Campinas: IMECC, v. 29, 1999. Disponível em: https://www.researchgate.net/ profile/Rodney_Bassanezi/publication/242289078_Modelagem_Matematica_Uma_disciplina_emergente_ nos_programas_de_formac_ao_de_professores/links/0c9605251e38583e5a000000.pdf. Acesso em: 3 fev. 2018.

CHAPRA, S. C.; RUNKEL, R. L. Modelling impact of storage zone on stream dissolved oxygen. J. Envoron. Eng. [S.I. s.n.], v. 125, n. 5, p. 415-419. 1999.

CUNHA, A. C.; CONEGHIAN, C. M. R.; POLETTI, E. C. C. Sewage discharge and water self decay: Streeter and Phelps model application. Computational and applied mathematics. [S.I. s.n], v. 37, n. 3, p. 35143524, 2017.

DEKISSA, T.; MEIRLAEN, J.; ASHTON, P. J.; VANROLLEGHEM, P. A. Simplyfing dynamic river water quality modelling: a case study of inorganic nitrogen dynamics in the crocodile river (South Africa). Water, air and Soil Pollution. [S.I. s.n], v. 155, p. 303-320, 2004.

ERTURK, A.; GUREL, M.; EKDAL, A.; TAVSAN, C.; UGURLUOGLU, A.; SEKER, D. Z.; TAMIK, A.; OZTURK, I. Water quality assessment and meta model development in Melen watershed - Turkey Journal of Environmental Management. [S.I. s.n], v. 91, p. 1526-1545, 2010. 
FAN, C.; KO, C. K.; WANG, W. S. An innovative modeling approach using QUAL 2K and HEC-RAS integration to assess the impact of tidal effect on river with quality simulation. Journal of Environmental Management. [S.I. s.n], v. 90, n. 5, p. 1824-1832, 2009.

GONÇALVES, J. C. S. I.; ESPOSTO, M. S. Water quality modelling of the São Joaquim stream, Brazil. Ciência e Natura. [S.I. s.n], v. 41, p. 1-13, 2019.

GOULART, M. D.; CALLISTO, Marcos. Bioindicadores de qualidade de água como ferramenta em estudos de impacto ambiental. Revista da FAPAM, [S.I. s.n], v. 2, n. 1, p. 156-164, 2003.

FRAGOSO JR, C. R; FERREIRA, T. F.; DA MOTTA MARQUES, D. Modelagem ecológica em ecossistemas aquáticos. [S.I.]: Oficina de textos, 2009.

GOMES, S. H. G.; GUEDES, H. S. A.; SIQUEIRA, T. M.; CORREA, L. B.; ANDREAZAA, R.; HUFFNER, A. N. Modelagem sazonal da qualidade de água do rio dos Sinos/RS utilizando o modelo QUAL-UFMG. Engenharia Sanitária e Ambiental. [S.I s.n], v. 23, p. 275-285, 2018.

GOTOVTSEV, A. V. Modification of the Streeter-Phelps system with aim to account for the feedback between dissolved oxygen conservation and organic matter oxidation rate. Water resources. [S.I. S.n], v. 37, n. 2, p. 245-251. 2010.

HAIDER, H.; AL, W.; HAYDAR, S. A rewiew of dissolved oxygen and biochemical oxygen demand models for large rivers. Pak. J. Eng. \& Appl. Sci. [S.I. s.n], v. 12, p. 127-142, 2013.

HOLANDA, V. P. D.; SOARES, T. R.; VERAS, I. F. O.; SALES, R. J. M. Análise do déficit e da concentração de oxigênio dissolvido no rio Piranhas-Açu, sujeito a lançamento de efluentes, mediante a utilização do modelo Streeter-Phelps simplificado. Rev. Tecnol. Fortaleza; Unifor, v. 38, n. 1, p. 55-66, 2017.

HUANG, B. H.; HIEN, H. N.; DINH, N. Y.N.; THAO, N. A.; HA, P.T.T.; KANDA SAMY, J. Integration of SWAT and QUAL $2 \mathrm{~K}$ for water quality modelling in a Delta scarce basin of can river basin in Vietnam. Ecohydrology \& Hidrobiology. [S.I.s.n] v. 19, p. 210-223, 2019.

JUBB, S.; HUKME, P.; GYMER, F.; MARTIN, J. Considering the impact of intermitent discharges when modeling overflows. Water science and technology. [S.I.s.n], v. 38, n. 10, p. 23-30. 1998.

LINDENSCHMIDT, K. E.; PECH, I,; BABOROWSKI, M. Environmental risk of dissolved oxygen depletion of diverted flood waters in river polder systems - A quasi $2 \mathrm{D}$ flood modeling approach. Science of the total environment. [S.I.s.n], v. 407, n. 5, p. 1598-1612. 2009.

LIU, Y.; SCAVIA, D. Analysis of the Chesapeake Bay hypoxia regime schift: insights from two simple mechanistic models. Estuaries and coasts. [S.I.s.n], v. 33, p. 629-639. 2010.

NAS, S. S.; NAS, E. Water quality modelling and dissolved oxygen balance in stream: a point source Streeter-Phelps application in the case of the Harsit stream. Clean. [S.I.s.n], v. 37, n. 1, p. 67-74, 2009.

MOTTA, D.; ABAD, J. D.; GARCIA, M. Modeling framework for organic sediment resuspension and oxygen demand: case of Bubbly creek in Chicago. J. Environ. Eng. [S.I s.n], v. 136, n. 9, p. 952-964, 2010.

McMARTIN, B. J.; FORRESTER, S. B. J. A fractional step-exponencially fitted hopscotch scheme for the Streeter-Phelps equations of river self-depuration. Engineering computation. [S.I s.n], v. 19, n. 2, p. 177189, 2002.

NUNES, D. G. Modelagem da autodepuração e qualidade da água do rio turvo sujo. Orientador: Demetrius David da Silva. 92f. 2008. Dissertação (Mestrado em Engenharia Agrícola). Universidade Federal de Viçosa, Viçosa, 2008. 
OMOLE, D.O.; LONGE, E. O. Reareation coefficiente modelling: a case study river Atuwara in Nigeria. Research Journal of applied sciences engineering and technology. [S.I s.n], v. 4, n. 10, p. 1237-1243, 2012.

ORSSATTO, F. Avaliação do oxigênio dissolvido do Córrego Bezerra a montante e a jusante de uma estação de tratamento de esgoto sanitário, Cascavel, Paraná. Revista Brasileira de Biociências. Porto Alegre: Instituto de Biociências do Rio Grande do Sul, v. 6, 2008. Supl. 1.

RAUCH, W.; HENZE, M.; KONCSOS, L.; REICHRT, P. River water quality modelling I. State of the art. Water and technology. [S.I s.n], v. 38, n. 11, p. 237-244, 1998.

RICCIARDONE, P.; DOS SANTOS PEREIRA, O.; PEREIRA, C. S. S. Avaliação da Capacidade de Autodepuração do Rio das Mortes no Município de Vassouras/RJ. Revista Eletrônica Teccen. Vassouras, v. 4, n. 3, p. 63-76, 2011.

RHODEN, A. C.; FELDMANN, N. A; MUHL, F. R.; RITTER, A. F. S.; MOREIRA, A. A importância da água e da gestão dos recursos hídricos. Revista de Ciências Agroveterinárias e Alimentos. [S./ s.n], n.1, p. 2016.

STRAGER, M. P.; FLETCHER, J. J.; STRAGER, J. M.; YUILL, C.B.; ELLI, R. N.; PETTY, J. T.; LAMONT, S. J. Watershed analysis with GIS: The watershed characterization and modelling system software application. Computer \& Geoscience. [S.I s.n], v. 36, p. 970-976, 2010.

STREETER, H. W.; PHELPS, Earle B. A study of the pollution and natural purification of the Ohio River, III, factors concerned in the phenomena of oxidation and reaeration. Public Health Bulletin. Washington: US Public Health Service, 1958. n. 146.

SODRÉ, U. Modelos matemáticos. Londrina: Universidade Estadual de Londrina, 2007. Disponível em: http://www.uel.br/projetos/matessencial/superior/pdfs/modelos.pdf. Acesso em: 3 fev. 2018.

TEODORO, A.; Ide, C. N.; Ribeiro, M. L; Broch, S. A. O.; Silva, J. D. Implementação do conceito capacidade de diluição de efluentes no modelo de qualidade da água QUAL-UFMG: estudo de caso no Rio Taquarizinho (MS). Engenharia Sanitária e Ambiental. Rio de Janeiro, v. 18, n. 3, p. 275-288, jul./set. 2013.

TONON, K. Modelagem da qualidade da água utilizando os modelos Streeter-Phelps e QUAL-UFMG na Bacia do Rio Lambari-Poços de Caldas. Orientador: Antônio Donizetti Gonçalves de Souza. 2014. 167f. Dissertação (Mestrado em Ciência e Engenharia Ambiental) - Universidade Federal de Alfenas, Poços de Caldas, 2014.

TSUKAMOTO FILHO, A. T.; CAMPOS, M. N.; VASCONCELOS, L. M. R.; MATOS, S. P. Diversidade florística de um parque zoobotânico no município de Santarém-Pará. In: CONGRESSO DE ECOLOGIA DO BRASIL, 8. 2007. Caxambu. Anais [...]. Caxambu: Sociedade de Ecologia do Brasil, 2007.

TUNDISI, J. G. Água no século XXI: enfrentando a escassez. São Carlos: RiMa, 2003, 248p.

VON SPERLING, M. Introdução à qualidade das águas e ao tratamento de esgotos. Belo Horizonte: Editora UFMG, 2005.

VON SPERLING, Marcos. Estudos e modelagem da qualidade da água de rios. 2. ed. Belo Horizonte: Editora UFMG, 2014.

WAGHMADE, R. W.; KIWNE, S. B. Matematical modelling for dissolved oxygen SAG analysis in river. International Journal of Engineering Science and Mathematics. [S.I s.n], v. 8, n. 2, p. 38-47, 2019. 
WANG, L. K.; VIELKIND, D.; WANG, M. H. Mathematical models of dissolved oxygen concentration on fresh water. Ecological Modelling. [S.I s.n], v. 5, n. 2, p. 115-123, 1978.

YUSTIANI, Y. M.; LIDYA, L.; BANDUNG, I. Towards an informative system of modeling and monitoring of Cikapundung river. Procedia Engineering. [S.I s.n], v. 154, p. 353-360, 2016.

YUSTIANI, Y. M. Determination of maximun BOD load using water quality modelling of upstream Citarum river. International Journal of GEOAMATE. [S.I S.n], v. 16, n. 56, p. 118-122, 2019.

ZOPPAS, F. M.; BERNARDES, A. M.; MENEGUZZI, A. Parâmetros operacionais na remoção biológica de nitrogênio de águas por nitrificação e desnitrificação simultânea. Engenharia Sanitária e Ambiental. [S.I s.n], v. 21, n. 1, p. 29-42, 2016.

\section{Sobre os autores}

\section{Raquel Freitas dos Santos}

Graduação em Ciências e Tecnologia das Águas pela Universidade federal do Oeste do Pará (2018). Atualmente cursando Bacharelado em Engenharia Sanitária e Ambiental pela Universidade federal do Oeste do Pará. Petiana do Programa de Educação Tutorial - PET Conexões de Saberes de Estudos Interdisciplinares: Comunidade do Campo (Ufopa).

\section{Zaqueu dos Santos}

Graduação em Ciências e Tecnologia das Águas pela Universidade federal do Oeste do Pará (2018). Atualmente cursando Bacharelado em Ciências Biológicas pela Universidade federal do Oeste do Pará.

\section{Mirian Santos de Sousa}

Graduação em Ciências e Tecnologia das Águas pela Universidade federal do Oeste do Pará (2018). Atualmente cursando Bacharelado em Engenharia Sanitária e Ambiental pela Universidade federal do Oeste do Pará.

\section{Ruy Bessa Lopes}

Pós-Doutorado em Ecotoxicologia pelo CENA/USP Universidade de São Paulo (2010). Doutorado em Ciências (energia nuclear na agricultura) pela Universidade de São Paulo (2005). Mestrado em Produção Animal pela Universidade Estadual de São Paulo (2000). Tem experiência em ecologia aplicada com ênfase na ecotoxicologia de moléculas orgânicas e poluição aquática. Biólogo e atualmente professor da Universidade Federal do Oeste do Pará, coordenador do curso de Engenharia Sanitária e Ambiental da Universidade Federal do Oeste do Pará.

\section{Lucinewton Silva de Moura}

Pós-doutorado na Faculdade de Engenharia Química pela Universidade Federal do Pará (2005). Doutorado em Engenharia de Alimentos com ênfase em desenvolvimentos de processos pela Universidade Estadual de Campinas (2004). Mestrado em Engenharia Química pela Universidade Federal do Pará (2000). Graduação em Engenharia Química pela Universidade Federal do Pará (1996). É professor associado III da Universidade Federal do Oeste do Pará (Ufopa), lotado no Instituto de Ciências e Tecnologia das Águas (ICTA) no Bacharelado em Engenharia Sanitária e Ambiental. Atualmente, é diretor do Instituto de Ciências e Tecnologia das Águas - ICTA da Universidade Federal do Oeste do Pará. Foi coordenador do Bacharelado em Engenharia Sanitária e Ambiental no biênio 2016-2018. Possui larga experiência na coordenação de cursos de graduação e de projetos de pesquisa e de extensão. É membro de um grupo de pesquisa que tem desenvolvido vários projetos de pesquisa, ao longo dos últimos anos, relacionado com o grau de comprometimento dos recursos hídricos da Amazônia, objetivando identificar danos ao meio ambiente através de ações integradoras (aspectos bioecológicos, de hidrologia e geomorfológicos, combinados aos aspectos socioambientais). Foi coordenador do curso de Engenharia Sanitária e Ambiental/ICTA no biênio 2012 a 2014. Presidiu o núcleo docente estruturante do curso de Engenharia Sanitária e Ambiental/ICTA pela Universidade Federal do Oeste do Pará de 2012 a 2014. Foi diretor da Faculdade de Engenharia de Minas e Meio Ambiente da Universidade Federal do Pará, onde fez parte da equipe de implantação do curso e da reformulação no projeto pedagógico. Atua nas áreas: química de águas continentais, química aplicada a saneamento ambiental e recursos hídricos, modelagem matemática de recursos hídricos.

Recebido em: 28.11.2019

Aceito em: 21.03.2020 